\title{
Study on the Pyrolysis Kinetics of Cotton Stalk based on the Multiscanning Method
}

\author{
L. ZHOU, L.Y. WANG, F. LIU, J.L. SUN, Z.Y. LI, C.L. QIN \\ School of Mechanical and Electrical Engineering, Tarim University, Alar Xinjiang 843300 \\ The Key Laboratory of Colleges \& Universities under the Department of Education of Xinjiang Uygur \\ Autonomous Region, Alar Xinjiang 843300
}

\begin{abstract}
Cotton stalk pyrolysis behavior and mechanism were studied based on the multi-scanning method. The results showed that cotton stalk pyrolysis zone during 30 600 ${ }^{\circ} \mathrm{C}$ could be divided into four regions, namely, water loss, depolymerization "glass transition", main pyrolysis and charring stage. During the main reaction zone TG curves and differential TG curves were moving to high temperature. The activation energy $\mathrm{E}$ of the main stage of pyrolysis was solved by FWO and FRL method respectively. The results showed that the activation energy $\mathrm{E}$ was very stable in the conversion rate of $10 \%$ to $70 \%$. The results of the two solving methods were very close and had a high degree of linear fitting. Experimental data were analyzed by the non-linear fitting, and the mechanism of cotton stalk pyrolysis met Avrami-Erofeev equation. The reaction value of series (grade) was 0.4. The mechanism function forms are $G(\alpha)=[-\ln (1-\alpha)]^{\frac{2}{3}}$ and $f(\alpha)=\frac{5}{2}(1-\alpha)[-\ln (1-\alpha)]^{\frac{3}{5}} \quad$ respectively.
\end{abstract}

KEYWORD: Cotton Stalk; Multi-scanning Method; Pyrolysis Mechanism; Dynamics

\section{INTRODUCTION}

Xinjiang is Chinese largest base for cotton production and cotton production accounts for about $1 / 3$ in China. While the cotton is harvested, that will produce large amounts of waste cotton stalk, cottonseed, cottonseed hulls and so on. According to statistics, Xinjiang produces cotton stalk for 600 7500000t (wet material) per year, but the utilization rate is very low, only partly used as low value fuel or feed, most returned field directly or burned in the field, which not only causes environmental pollution, but wastes resources. At present, the thermochemical treatment technology is one of the effective ways of realizing transform of agricultural by-products.

At present, foreign scholars have done amount of research on cotton waste pyrolysis (Vamvuka, E. Karakas et al. 2003, Badie S. Girgis \& Mona F. Ishak 1999, Cagiar, A \& Demirbas, A 2000, Ay Ye E. Pqtqn et al. 2005, Sehoon Kim \& Holtzapple M T 2006, Williams P T \& Nittaya Nugranad 2000). Our country mainly did some research on cotton stalk pyrolysis, reported Hu Song. Zhou Ling et al (2009) used three approaches that were Flynn-Wall-Ozawa method, double trendextrapolation method and Popescu method to carry on mechanism deduction, indicating that the pyrolysis process was controlled by the three-dimension diffusion mechanism function. Chen Dongyu \& Liu Ronghou (2007) studied cotton stalk pyrolysis behavior by the way of using alkaline catalyst, pickling and alkali washing cotton stalk, and the most probable mechanism function Avrami-Erofeev equation was more proper for cotton stalk pyrolysis process to be treated meeting, which was a random nucleation and later growth mechanism function, whose reaction order was 2 , but the most possible mechanism functions of cotton stalk untreated biomass pyrolysis met Zhuralev-Lesakin-Tempelman equation, which was a three-dimension diffusion mechanism function. Because of many factors affecting the biomassdynamics, such as the types of material, temperature, experimental method and so on, getting pyrolysis mechanism was also not consistent. In order to reflect the pyrolysis process of cotton stalk truly, this paper was based on the activation energy E solved by the the multi-scanning method of cotton stalk and certainty of mechanism function, providing theoretical basis for utilization of cotton stalk. 
2 THE EXPERIMENTAL PART

\subsection{Raw materials}

Experimental cotton stalk were from ccri35 experimental farm of Tarim University Xinjiang, making them dried and crushed, in order to reduce the influence of water content on the pyrolysis experiments, selection of the particle diameter size $<80$ items, the industrial analysis and component analysis shown in Table 1.

Table 1. Proximate analysis of cotton stalk

\begin{tabular}{|c|c|c|c|c|c|c|}
\hline Moisture Conmen \% & Ash \% & Volatile matter \% & Total cellulose \% & Pentosan \% & Lignin \% & Cellulose \% \\
\hline 14.147 & 3.942 & 65.604 & 73.24 & 18.8 & 21.0 & 42.8 \\
\hline
\end{tabular}

\subsection{The experimental method}

The experimental instrument used Netzsch STA449c thermogravimetric analyzer and the crucible for the domestic alumina crucible that was calcined at $1300^{\circ} \mathrm{C}$ before it was used. Each took about $8 \mathrm{mg}$ sample in the crucible, in order to reduce the influence of the heat and mass transfer and temperature gradient inside the material during pyrolysis process. Waiting for temperature was set at $30^{\circ} \mathrm{C}$ to ensure that each sample and baseline started with the initial temperature of $30^{\circ} \mathrm{C}$ and reduce errors that resulted from the difference of initial rise temperature. The pyrolysis temperature range in the experiment was $30^{\circ} \mathrm{C} \sim 600^{\circ} \mathrm{C}$, the heating rates were set at $5,10,20,30 \mathrm{~K} / \mathrm{min}$, using $99.99 \%$ nitrogen as protective gas, flow rates set at $30 \mathrm{ml} / \mathrm{min}$.

\section{ANALYSIS AND DISCUSSION OF EXPERIMENTAL RESULTS}

As shown in Figure 1, at 4 different heating rates, the pyrolysis trend of TG-DTG curves were similar, only TG curves and DTG curves moving to high temperature. The initial temperature, termination temperature, weight loss rate and the peak point of weightlessness of reaction had a slight difference, showing that with the increase of heating rates, the pyrolysis mechanism of each sample did not change. the DTG curves showed that maximum pyrolysis rate and its corresponding temperature increased with the increase of heating rates, temperature range of pyrolysis expanding; TG curves showed that the required temperature of reaching the same weight loss rate increased a little.

Pyrolysis curves of cotton stalk could be divided into four regions: the temperature range of $31 \sim 140^{\circ} \mathrm{C}$ as the first area, namely, water loss stage, water loss rate of about $6 \%$, and TG curves showed a smaller weight loss. At this stage, the DTG curves showed a smaller loss rate; the temperature range of $141 \sim 207^{\circ} \mathrm{C}$ as the second area, namely, process of "glass transition". Both TG curves and DTG curves tended to flat. At this stage, depolymerization and polymerization occured in the internal structure of cotton stalk, shaping the free radicals and the main functional groups, releasing a small amount of small molecular volatile gas; temperature range of 208 $368^{\circ} \mathrm{C}$ as the third district that was also main pyrolysis stage of cotton stalks, weight loss rate of $53 \%$, the sample had weight loss, the TG curve showed a sharp decline, dynamics analysis of cotton stalk mainly aimed at this stage; the temperature range of $369 \sim 600^{\circ} \mathrm{C}$ as the fourth district that was carbonization stage, slow decomposition process of residues, the main formation of char and ash, this moment the TG curves and DTG curves tended to flat.

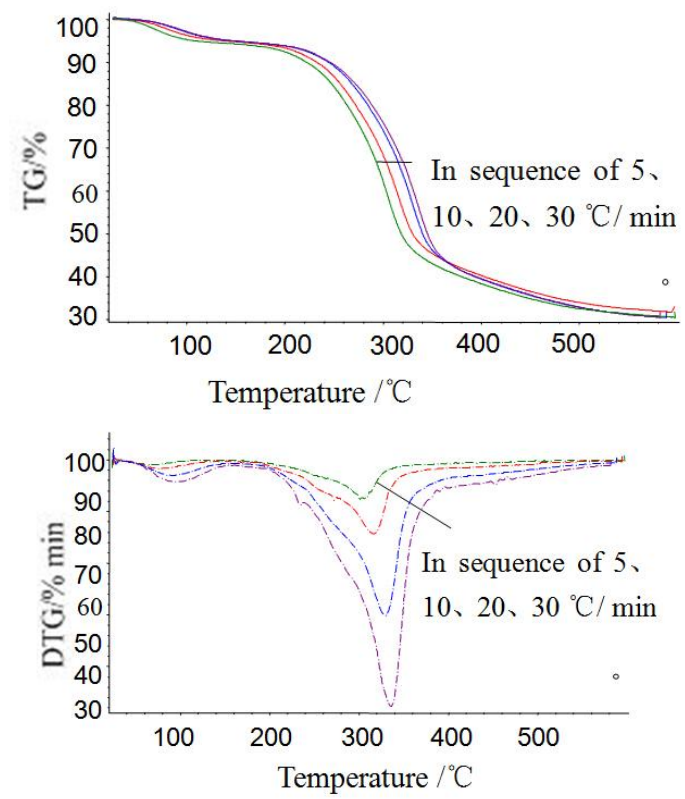

Fig.1. Part pyrolysis curves of cotton stalk at different heating rates

Table 2. Characteristic parameters of pyrolysis at different heating rates

\begin{tabular}{|c|c|c|c|c|}
\hline$\beta /{ }^{\circ} \mathrm{C} * \min ^{-1}$ & $\mathrm{~T}_{\max } /{ }^{\circ} \mathrm{C}$ & $\mathrm{T}_{\max } /{ }^{\circ} \mathrm{C}$ & $\mathrm{W}_{\mathrm{f}} / \%$ & $\mathrm{~T}_{\mathrm{e}} \sim \mathrm{T}_{\mathrm{f}} /{ }^{\circ} \mathrm{C}$ \\
\hline 5 & 302 & 3.8 & 69.57 & $260.2 \sim 327.8$ \\
\hline 10 & 316.8 & 7.18 & 68.36 & $266.2 \sim 337.7$ \\
\hline 20 & 329.8 & 15.07 & 69.64 & $278.2 \sim 351.8$ \\
\hline 30 & 336.4 & 23.88 & 69.62 & $285.4 \sim 356.4$ \\
\hline
\end{tabular}

Note: $\beta$ - heating rates; $\mathrm{T}_{\max }$ - the peak temperature; $(\mathrm{d} \alpha / \mathrm{dt})_{\max }-$ maximum pyrolysis rates; $\mathrm{W}_{\mathrm{f}}$-the final weight loss rate; $\mathrm{T}_{\mathrm{f}}-$ the finish temperature of main pyrolysis; $\mathrm{T}_{\mathrm{e}}-$ the initial temperature of main pyrolysis. 
4 THE DEDUCTION OF MECHANISM FUNCTION AND CALCULATION OF KINETIC PARAMETERS

\subsection{Calculation of the activation energy}

The thermal stability of the biomass pyrolysis could be characterized by the activation energy $\mathrm{E}$ that was important kinetics parameters. There were many methods that the traditional rate equation calculated the $\mathrm{E}$ value, but at present a more reliable method recognized was multi-heating rate methods (STARINK M J 2003), and its essence was to calculate the $\mathrm{E}$ value with a plurality of $\mathrm{TG}$ measured in different heating rates, one of which was using $\alpha$ value that come from the same temperature of different TG curves, called isoconversional method. The advantage of this method was to obtain more reliable $\mathrm{E}$ values in unknown mechanism functions, in addition, whether reaction mechanism in the whole pyrolysis process changes or not was studied by comparing $E$ values in different $\alpha$. In recent years, this kind of method gets more and more attention.

\subsubsection{Calculation of the activation energy $E$ with Ozawa method (OZAWAT 1965)}

Since the Ozawa method did not involve the form of mechanism function, to avoid errors that different reaction mechanisms bring, so the activation energy E calculated was used to test the activation energy that was calculated by single scanning rate method.

$$
\log \beta=\log \left(\frac{A E}{R G(\alpha)}\right)-2.315-0.4567 \frac{E}{R T}
$$

Where $\mathrm{A}$-freqency factor, $\mathrm{s}^{-1} ; \beta$-heating rate, $\quad{ }^{\circ} \mathrm{C} / \mathrm{min} ; \mathrm{R}$-universal gas constant, $8.314 \mathrm{~J} /(\mathrm{kg} \cdot \mathrm{K})$; E-activation energy, $\mathrm{kJ} / \mathrm{mol}$; $\mathrm{T}$ - the reaction temperature $(\mathrm{K})$; and $G(\alpha)$-integral form of reaction mechanism function.

At the equivalent conversion rate, $\log \beta$ was linear with $\frac{1}{T}$, and mapping the two could get a straight line. By the slope of the line could solve the reaction activation energy.

\subsubsection{Calculation of the activation energy $E$ with Friedman-Reich-Levi method}

The differential form of pyrolysis equation was organized into the following form:

$$
\ln \left[\frac{\beta d \alpha}{d T}\right]=\ln A f(\alpha)-\frac{E}{R T}
$$

Where $\alpha, \quad \ln A f(\alpha)$ was a constant, and $\ln \left[\frac{\beta d \alpha}{d T}\right]$ was linear with $\frac{1}{T}$. By the slope of the line could solve the reaction activation energy.

\subsubsection{Results and analysis of calculating activation energy with two methods}

Since the FWO method did not involve the form of mechanism function, to avoid errors that different reaction mechanisms bring, so the activation energy E calculated was used to test the activation energy that was calculated by the other assumption reaction mechanism function. Because conversion rate of the FRL method in value was very sensitive to selection of baseline and recording precision of thermal analyzer, therefore, experimental conditions were controlled preciously, and E value with Friedman method could reflect the process of biomass pyrolysis. Numerical calculation of the above two methods had the same difficulty. This paper calculated the activation energy with this two method and obtained reliable pyrolysis kinetic parameters of cotton stalk by comparing kinetic parameters, height of linearity and the difference between the two methods. In the Ozawa method and Friedman-Reich-Levi method, if reaction excitation function was known only, the frequency factor A could be obtained. On the assumption that the cotton stalk pyrolysis met: $f(\alpha)=1-\alpha$ and $G(\alpha)=\ln (1-\alpha)$, could be calculated approximately the size of $\mathrm{A}$. In this paper, the conversion rate $\alpha$ was taken as $10 \%$ to $90 \%$. Using the formula of FWO (1) formula and FRL method (2) calculated the activation energy. As could be seen from table 3, when the conversion rate was higher than $80 \%$, the activation energy solved increased rapidly, which was a drawback of iso-conversional method. In the range of $10 \%$ to $70 \%$ of the conversion rate, the activation energy in the main pyrolysis stage of cotton stalk was very stable, the $\mathrm{E}(\mathrm{FWO})=181.71 \pm 2.2 \mathrm{~kJ} / \mathrm{mol}, \mathrm{E}(\mathrm{FRL})=181.68 \pm 1.5$ $\mathrm{kJ} / \mathrm{mol}$, and the approximate solution of $\lg \mathrm{A}$ was about $\lg \mathrm{A} \quad(\mathrm{FWO})=14.7, \lg \mathrm{A} \quad(\mathrm{FRL})=14.5$. The results of the two solving methods were very close and had a high degree of linear fitting, illustrating that the activation energy soved might reflect the cotton stalk pyrolysis behavior truly. 
Table 3. Activation energy and correlation coefficient from the method of FWO and FRL

\begin{tabular}{|r|c|c|c|c|c|c|}
\hline$\alpha \%$ & $\mathrm{E}(\mathrm{FWO}) / \mathrm{kJ} / \mathrm{mol}$ & $\lg$ (FWO) & Rsq(FWO) & $\mathrm{E}(\mathrm{FRL}) / \mathrm{kJ} / \mathrm{mol}$ & $\operatorname{lgA}(\mathrm{FRL})$ & Rsq(FRL) \\
\hline 10 & 181.86 & 15.65 & 0.988 & 181.5 & 15.24 & 0.986 \\
\hline 20 & 179.73 & 14.98 & 0.993 & 180.22 & 14.64 & 0.996 \\
\hline 30 & 180.33 & 14.72 & 0.995 & 181.95 & 14.45 & 0.994 \\
\hline 40 & 182.20 & 14.64 & 0.996 & 182.24 & 14.22 & 0.986 \\
\hline 50 & 182.07 & 14.43 & 0.998 & 181.44 & 14.01 & 0.991 \\
\hline 60 & 181.82 & 14.27 & 0.996 & 181.46 & 13.95 & 0.992 \\
\hline 70 & 183.93 & 14.36 & 0.992 & 182.95 & 15.01 & 0.996 \\
\hline 80 & 195.58 & 15.28 & 0.996 & 198.07 & 17.85 & 0.999 \\
\hline 90 & 257.42 & 20.30 & 0.992 & 229.23 & 25.19 & 0.979 \\
\hline
\end{tabular}

\subsection{The deduction of mechanism function}

TG experimental data was analyzed by the nonlinear fitting with Netzsch thermodynamic analysis software, and investigate the adaptability of experimental data that come from different mechanism function. Taking 41 mechanism functions (Hu Rong \& Shi Qizhen 2001) to nonconstant temperature pyrolysis equation $\frac{d \alpha}{d t}=\left(\frac{A}{\beta}\right) \exp \left(-\frac{E}{R T}\right) f(\alpha)$, the fitting results, size of sum of square of difference value between experimental data and the regression coefficient as the measure of selecting mechanism function, after fitting , obtaining kinetic parameters were shown in table 4, and table 4 only listed fitting results of the four kinds of mechanism function. Comparing the kinetic parameters that were calculated with different mechanism function with the above results, calculation result of mechanism function of An form was very close to the above results, which could determine the mechanism of cotton stalk pyrolysis met Avrami-Erofeev equation, namely random nucleation and later growth mechanism function, reaction order approximated $\mathrm{n}=0.4$, and the mechanism function forms are $G(\alpha)=[-\ln (1-\alpha)]^{\frac{2}{3}}$, $f(\alpha)=\frac{5}{2}(1-\alpha)[-\ln (1-\alpha)]^{\frac{3}{5}}$, the fitting result shown in figure 2. Physical meaning of the mechanism function showed cotton stalk pyrolysis process that because of reactivity of cotton stalk with different components caused complexity, and when the reaction order was not equal to 1 , it indicated that the reaction was anisotropic, then both the reaction area and diffusion affecting process of the reaction. In the initial stage of the reaction, the phase in some local point produced active center randomly.

With generation of the new active center, a part of the active center leads to inactivation or decomposition. Both the fitting results and mechanism function that Chen Dongyu obtained after cotton stalk was pretreated belong to An, but the pyrolysis reaction orders had some difference.

Table 4. Kinetic results calculated by fitting curve

\begin{tabular}{|c|c|c|c|c|}
\hline Function form & $\operatorname{lgA}$ & $\mathrm{E}$ & $\mathrm{n}$ & $\mathrm{Rsq}$ \\
\hline An & 14.48 & 182.82 & 0.4430 & 0.998 \\
\hline D3 & 10.14 & 147.58 & - & 0.998 \\
\hline Fn & 9.83 & 128.88 & 2.14 & 0.989 \\
\hline R3 & 6.01 & 97.5 & - & 0.972 \\
\hline
\end{tabular}

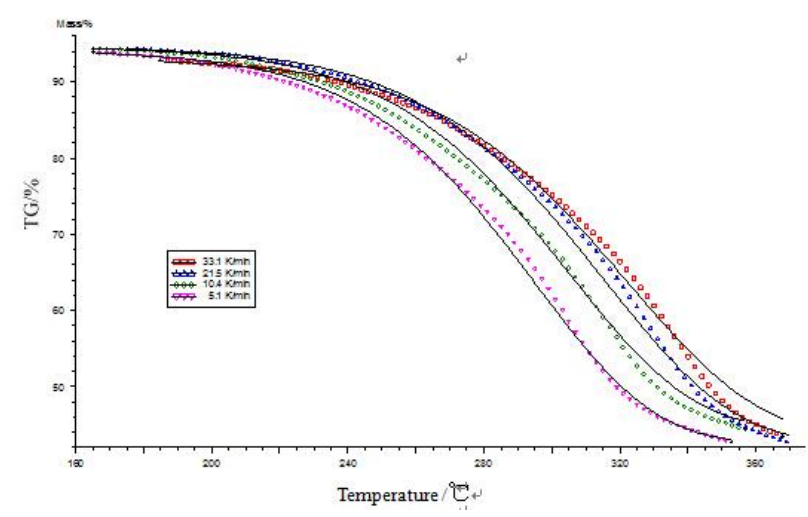

Fig.2. Compare of simulated data with experiment data

\section{CONCLUSION}

Cotton stalk pyrolysis behavior and mechanism were studied based on the multi-scanning method. The results showed that cotton stalk pyrolysis zone during 30 600 ${ }^{\circ} \mathrm{C}$ could be divided into four regions, namely, water loss, depolymerization "glass transition", main pyrolysis and charring stage. During the main reaction zone TGtivation energy E was solved by FWO and FRL method respectively. The results of the two solving methods were very close and had a high degree of linear fitting. The results showed that the activation energy $\mathrm{E}$ was very stable in the conversion rate of $10 \%$ to $70 \%$. Experimental data were analyzed by the non-linear fitting, $\quad \mathrm{E}(\mathrm{FWO})=181.71 \pm 2.2 \mathrm{~kJ} / \mathrm{mol}, \quad \mathrm{E}(\mathrm{FRL})=$ $181.68 \pm 1.5 \mathrm{~kJ} / \mathrm{mol}$. 
The mechanism pyrolysis function of cotton stalk was Avrami-Erofeev equation. The function mechanism forms are both curves and differential TG curves were moving to high temperature.

The mechanism function forms are $G(\alpha)=[-\ln (1-\alpha)]^{\frac{2}{3}}$ and $f(\alpha)=\frac{5}{2}(1-\alpha)[-\ln (1-\alpha)]^{\frac{3}{5}}$, namely random nucleation and later growth mechanism function, reaction order approximated $n=0.4$.

\section{REFERENCES}

[1] Ay Ye E. Pqtqn, Nurgql O” zbay, Eylem P. O” nal \& Ersan. 2005. Pqtqn Fixed-bed pyrolysis of cotton stalk for liquid and solid products. Fuel Processing Technology, 86:1207- 1219.

[2] Badie S. Girgis \& Mona F. Ishak. 1999. Activated carbon from cotton stalks by impregnation with phosphoric acid. Materials Letters, 39:107-114.

[3] Cagiar, A. \& Demirbas, A.2000. Conversion of cotton cocoon shell to liquid products by pyrolysis. Energy Convers. Manage. 41 (15):1749-1756.
[4] Chen Dongyu \& Liu Ronghou.2007. Study on the PyrolysisK inetics of Pre-treated Cotton Stalk. Transactions of the Chinese Society for Agricultural Machinery, 2007, 38(6):95-99.

[5] D. Vamvuka, E. Karakas, E. Kastanaki \& P. Grammelis. 2003. Pyrolysis characteristics and kinetics of biomass residuals mixtures with lignite. Fuel, 82: 1949- 1960.

[6] Hu Rongzu \& Shi Qizhen.2001. Thermal Analytic Kinetics. Beijing: Science Press.

[7] OZAWA T. 1965. A newmethod of analyzing thermogravimatric data. Bulletin of the Chemical Society of Japan, 38:1881-1886.

[8] Sehoon Kim \& Holtzapple M T.2006. Delignification kinetics of corn stover in lime pretreatment. Bioresource Technology, 97(5):778 785.

[9] Williams P T \& Nittaya Nugranad. 2000. Comparison of products from the pyrolysis and catalytic pyrolysis of rice husks. Energy, 25: 493 513.

[10] Yang Shuhui, Li Yousen \& Gao Yang. 1989. The Research and Characterization of Cotton Stalk as Papermaking Raw Material. China Paper, 2:20-27.

[11] Zhou Ling, Zhou Fujun, Jiang Enchen, et al. 2009. Pyrolysis characteristics and dynamics of different components of cotton stalk. Transactions of the CSAE, 25(8):220-225. 